\title{
Carbonic anhydrase 9 immunohistochemistry as a tool to predict or validate germline and somatic VHL mutations in pheochromocytoma and paraganglioma - a retrospective and prospective study
}

\author{
Judith Favier $\mathbb{D}^{1} \cdot$ Tchao Meatchi $^{1,2} \cdot$ Estelle Robidel $^{1} \cdot$ Cécile Badoual $^{1,2} \cdot$ Mathilde Sibony $^{3,4} \cdot$ An Thach Nguyen $^{2}$. \\ Anne-Paule Gimenez-Roqueplo ${ }^{1,5,6} \cdot$ Nelly Burnichon ${ }^{1,5}$
}

Received: 15 February 2019 / Revised: 22 July 2019 / Accepted: 23 July 2019 / Published online: 5 August 2019

(c) United States \& Canadian Academy of Pathology 2019

\begin{abstract}
The development of pheochromocytomas and paragangliomas is strongly linked to the presence of germline mutations in more than 15 predisposing genes. Among them, germline and somatic $V H L$ mutations account for $\sim 10 \%$ of all cases. In contrast with SDHA and SDHB immunohistochemistries that are routinely used to validate SDHx gene mutations, there is no such tool available for $V H L$ mutations. The aim of this study was to evaluate whether CA9 immunostaining could be used as a tool to predict the presence or validate the pathogenicity of $V H L$ gene mutations in paraganglioma. Immunohistochemistry for CA9 was performed on 207 tumors. A retrospective series of 100 paragangliomas with known mutation status for paraganglioma susceptibility genes was first investigated. Then, a prospective series of 107 paragangliomas was investigated for CA9 immunostaining followed by germline and/or somatic genetic testing of all paraganglioma susceptibility genes by next-generation sequencing. Cytosolic CA9 protein expression was heterogeneous in the different samples. However, we observed that a membranous CA9 staining was almost exclusively observed in VHL-related cases. Forty two of 48 (88\%) VHL-mutated samples showed a CA9 membranous immunostaining. Positive cells were either isolated, varying from 1 or 2 cells (5\% of cases) to $10-20$ cells per tumor block (35\% of cases), grouped in areas of focal positivity representing between 1 and $20 \%$ of the tissue section (35\% of cases), or widely distributed on $80-100 \%$ of the tumor sections ( $25 \%$ of samples). In contrast, 142/159 (91\%) of non-VHL-mutated tumors presented no membrane CA9 localization. Our results demonstrate that VHL gene mutations can be predicted or validated reliably by an easy-to-perform and low-cost immunohistochemical procedure. CA9 immunohistochemistry on paragangliomas will improve the diagnosis of VHL-related disease, which is important for the surveillance and therapeutic management of paraganglioma patients, and in case of germline mutation, their family members.
\end{abstract}

Supplementary information The online version of this article (https:// doi.org/10.1038/s41379-019-0343-4) contains supplementary material, which is available to authorized users.

Judith Favier

judith.favier@inserm.fr

1 Université de Paris, PARCC, INSERM, Equipe Labellisée par la Ligue contre le Cancer, F-75015 Paris, France

2 Assistance Publique-Hôpitaux de Paris (AP-HP), Hôpital Européen Georges Pompidou, Département d'anatomo-pathologie, F-75015 Paris, France

3 Assistance Publique-Hôpitaux de Paris, Hôpital Cochin, Service

\section{Introduction}

Paragangliomas are rare neuroendocrine tumors that arise in the sympathetic or parasympathetic nervous systems, from the head and neck, to the pelvic region. Pheochromocytomas are

d'anatomie-pathologie, F-75014 Paris, France

4 Université de Paris, F-75015 Paris, France

5 Assistance Publique-Hôpitaux de Paris (AP-HP), Hôpital Européen Georges Pompidou, Service de Génétique, F-75015 Paris, France

6 Rare Adrenal Cancer Network-Cortico Médullosurrénale Tumeur Endocrine, Institut National du Cancer, F-75014 Paris, France 
paragangliomas that develop in the adrenal medulla. Their occurrence is strongly linked to the presence of germline mutations in predisposing genes, and it is estimated that around $40 \%$ of all paragangliomas are caused by such a genetic defect. The major cancer syndromes that include paragangliomas in their tumor spectrum are neurofibromatosis type 1 (NF1 gene mutations), multiple endocrine neoplasia type 2 (RET mutations), von Hippel-Lindau disease (VHL mutations), or hereditary paraganglioma syndrome ( $\mathrm{SDHx}$ gene mutations) (for review see refs. [1, 2]). In addition, TMEM127, MAX, $\mathrm{FH}, \mathrm{MDH} 2, \mathrm{GOT2}$, DNMT3A, or SLC25A11 germline mutations are responsible for isolated or bilateral forms of paraganglioma [3-6].

It is considered that at least $10 \%$ of all paragangliomas carry either a germline or a somatic mutation in the VHL gene [7, 8]. Germline $V H L$ mutations predispose to the development of von Hippel-Lindau disease manifestations, including retinal and central nervous system hemangioblastomas, pancreatic neuroendocrine tumors, clear cell renal cell carcinomas, and paragangliomas. The wide age range and pleiotropic manner in which VHL disease presents, complicates diagnosis and treatment in affected individuals, as well as their at-risk relatives. Moreover, the presence of $V H L$ somatic mutations within the tumors may also be of clinical importance, as it is now suspected that $V H L$ - (and SDHx-) mutated patients may better respond to antiangiogenic therapies such as sunitinib [9, 10]. In that context, identifying the germline or somatic driver mutation in paragangliomas may be of interest in a precision medicine approach.

Because of the multiplicity of genes implicated in paraganglioma development and of the large number of variants of unknown significance now identified by nextgeneration sequencing methods, it has become a major challenge for pathology departments to be able to orientate the genetic test or to assess the pathogenicity of variants of unknown significance, using simple immunohistological tools. We have previously reported that SDHB [11], SDHA [12, 13], and SDHD [14] immunostaining can be efficiently used to guide genotyping toward $S D H x$ genes or validate the functionality of $S D H x$ variants in paraganglioma patients. SDHB and SDHA immunohistochemistries have been validated in large multicentric studies and are used routinely in pathology departments in charge of paraganglioma diagnosis worldwide [15]. $\mathrm{FH}$ mutations can be validated by 2-SC immunohistochemistry [16], while MAX immunostaining was shown to be negative in tumors carrying truncating $M A X$ mutations, but lacks sensitivity and specificity in tumors carrying missense MAX mutations [17]. A few studies have reported some markers that may be associated with the VHLmutated status such as EPO [18], clusterin [19], or carbonic anhydrase 9 (CA9) [20]. However, these studies were performed in a very small number of $V H L$ cases (between 1 and 5) and were never reproduced.

The aim of the current study was therefore to identify and validate an immunohistochemical marker that could be used to suspect $V H L$ gene inactivation or assess the pathogenicity of $V H L$ variants in paraganglioma patients. Because CA9 is a known hypoxia-induced gene and is overexpressed in clear cell renal cell carcinomas [21] that often carry (somatic) VHL gene mutations, we evaluated its expression in retrospective and prospective series of paragangliomas.

\section{Patients and methods}

\section{Patients}

The analysis of the transcriptome was carried out on the data from 188 paragangliomas, collected by the French "Cortico et Médullosurrénale: les Tumeurs Endocrines" (COMETE) network and previously published and described [22, 23]. The data sets are available in the following repository: ArrayExpress entry E-MTAB-733.

The retrospective study was based on a first series of 111 paraganglioma tumors from 109 patients, which included 81 pheochromocytomas, 17 sympathetic paragangliomas, 4 parasympathetic paragangliomas, 4 metastases, and 5 of unknown location collected by the French COMETE network from patients operated in two referral centers in Paris (Hôpital Européen Georges Pompidou and Hôpital Cochin) between 1977 and 2010. That series contained tumors with different germline or somatic mutations, including $26 \mathrm{VHL}$ (18 germline and 8 somatic cases), 17 SDHx (2 SDHA, 8 SDHB, 2 SDHC, 5 SDHD, and all germline), 13 NF1 (5 germline and 8 somatic cases), 9 RET (4 germline and 5 somatic cases), 4 MAX (2 germline and 2 somatic cases), 1 TMEM127 (germline), 1 FH/ATRX (respectively germline/ somatic), 1 SLC25A11 (germline), 2 HRAS (somatic mutations), 3 EPASl (somatic), 1 ATRX (somatic), and 33 apparently sporadic cases with no mutation identified (Supplementary Table 1a). The procedures used for paraganglioma diagnosis and genetic testing were in accordance with institutional guidelines. In 80/111 cases, tumor DNA was analyzed by next-generation sequencing using a custom paraganglioma gene panel, including $V H L, N F 1, R E T$, SDHA, SDHB, SDHC, SDHD, SDHAF2, TMEM127, FH, MAX, EPAS1, EGLN1, EGLN2, MDH2, ATRX, and HRAS genes ("MASTR Plus SDHv2" panel, Multiplicom, Agilent Technologies [24]). In other cases, mutation analysis for major paraganglioma susceptibility genes was performed by Sanger sequencing of germline DNA.

The prospective study was performed on 107 consecutive tumors from 107 patients, which were followed at the Hôpital Européen Georges Pompidou in Paris, or 
in different referral centers of the COMETE network in France, between December 2012 and July 2018 (Supplementary Table $1 \mathrm{~b}$ ). That second series comprised 54 pheochromocytomas, 42 sympathetic paragangliomas, and 11 parasympathetic paragangliomas. Genetic testing was performed after CA9 immunohistochemistry, using nextgeneration sequencing paraganglioma gene panel on both germline and tumor DNAs in 45 patients and on germline DNA only in 41 patients. Genetic screening was done using Sanger and MLPA for 21 patients, completed with nextgeneration sequencing on tumor DNA in six patients.

\section{Immunohistochemistry}

Four- to six-micrometer sections of formalin-fixed and paraffin-embedded archival tissues were cut and mounted on Superfrost plus glass slides.

CA9 immunohistochemistry was performed as follows, using the anti-CA9 antibody (ab 15086, abcam) in all cases. For the retrospective study, immunohistochemistry was performed manually. Following deparaffinization and rehydration, heat-mediated antigen retrieval was performed using Tris-EDTA at $\mathrm{pH} 9$ for $45 \mathrm{~min}$ followed by $\mathrm{H}_{2} \mathrm{O}_{2}$ treatment (3\%, 30 min). After blockade of unspecific sites in $10 \%$ normal goat serum for $30 \mathrm{~min}$, anti-CA9 antibody was applied (1:1500) for $1 \mathrm{~h}$ at room temperature in normal goat serum $1 \%$ followed by the biotinylated secondary anti-rabbit antibody (Vector Lab; 1:400) for 45 min. Amplification was performed with the avidin-biotin complex (ABC Elite, Vector Laboratories EUROBIO/ ABCYS, Les Ulis, France) for $45 \mathrm{~min}$ and the revelation was assessed using the histogreen kit (Vector Laboratories, EUROBIO/ABCYS, Les Ulis, France). Nuclear Fast Red counterstaining was performed before rehydrating and mounting of slides in Eukitt media (Sigma-Aldrich). Negative control was performed by omitting the primary antibody.

For the prospective study, immunohistochemistry was performed in the Pathology department of Hôpital Européen Georges Pompidou on a BenchMark ULTRA IHC/ISHstaining system. Three-micron sections of formalin-fixed and paraffin-embedded tissues were cut and mounted on Leica Surgipath Apex slides. Immunohistochemistry was performed on a VENTANA Benchmark Ultra automat using the anti-CA9 polyclonal antibody (ab 15086, abcam). Deparaffination was performed with Ventana EZ Prep solution (Ventana) at $72{ }^{\circ} \mathrm{C}$ for $8 \mathrm{~min}$. Heat-mediated antigen retrieval was performed using citrate buffer Cell Conditioning $\mathrm{CC} 1$ at $\mathrm{pH} 8$ for $20 \mathrm{~min}$. Endogen peroxidase was blocked after $\mathrm{H}_{2} \mathrm{O}_{2}$ treatment (ultra View Inhibitor) (Ventana) for 4 min at $37^{\circ} \mathrm{C}$. The anti-CA9 was applied (1:500) for $32 \mathrm{~min}$. The biotinylated secondary anti-rabbit and antimouse antibody (ultraView Multimer, Roche Laboratories,
France) was then applied for $8 \mathrm{~min}$ and the revelation was assessed using DAB/ $/ \mathrm{H}_{2} \mathrm{O}_{2}$ (Roche Laboratories, France) for $48 \mathrm{~min}$. Hematoxylin nuclear counterstaining for $4 \mathrm{~min}$ and bluing reagent for $4 \mathrm{~min}$ was performed before rehydrating and slides were dehydrated and coverslipped in Pertex media (Histolab).

Acquisitions were performed using Leica DM400B microscope, with $\times 40$ objective.

Slides were analyzed blindly, by two independent observers (JF and ATN for the retrospective study, and JF and TM for the prospective study).

\section{Results}

\section{Retrospective study}

We first analyzed CA9 expression level in the transcriptomic data that we previously generated in 188 paragangliomas recruited by the COMETE network. Interestingly, we found that CA9 expression levels were very homogeneous between the different genotypes of tumors (Fig. S1 and Supplementary Table 2). In contrast, it was highly heterogeneous within the group of $V H L$-mutated tumors. These tumors could be divided into two groups, one named "VHL-low", which showed CA9 levels comparable with that of the other tumors, and one, "VHL-high", with high or very high expression levels. This difference in CA9 mRNA levels could be associated neither with any clinical criteria nor with the type of $V H L$ mutation.

Anyhow, we speculated that CA9 may also be differentially regulated at protein level and therefore analyzed its expression and localization by immunohistochemistry in a retrospective series of 111 paragangliomas. Eleven tumors showed no CA9 labeling and were excluded from the study. In the remaining 100 samples, CA9 protein expression was highly variable between tumors, with some tumors with an expression restricted to endothelial cells, some with very little labeling, and some with a widespread cytoplasmic staining. None of these observations was associated with a specific genetic background, tumor location or prognosis. In contrast, we observed that the presence of cells with a CA9 staining localized to the plasma membrane was almost exclusively restricted to tumors harboring $V H L$ gene mutations. This could be in a very few cells within the tumor sample, or in almost every tumor cells on the slide. Altogether, we observed a membrane CA9 immunostaining in 32 tumors: 24/25 VHL-mutated, four tumors with no mutation identified, two tumors with somatic ATRX mutations (one sporadic case and one associated with a germline $\mathrm{FH}$-mutation), one case with an NFl somatic mutation, and one with a $M A X$ somatic mutation. Interestingly, the only $V H L$-mutated tumor with no membrane CA9 staining (\#11) originated from a patient carrying a germline VHL mutation, 
for whom another tumor (\#12) showed a membrane CA9 immunostaining (Table 1).

In this retrospective study, the presence of a tumor membrane CA9 staining was strongly associated with $V H L$ somatic or germline mutations, with a $96 \%$ sensitivity and a $75 \%$ specificity (Table 2 ).

\section{Prospective study}

Following this result, we transferred CA9 immunostaining to routine clinical practice in our pathology department and studied its expression prospectively in 107 consecutive paragangliomas, in parallel to the paraganglioma genetic testing performed by the next-generation sequencing assay.

Table 1 Summary of the results obtained in the retrospective and prospective studies

\begin{tabular}{|c|c|c|c|c|}
\hline \multirow[b]{2}{*}{ Mutated gene } & \multicolumn{2}{|l|}{ Retrospective } & \multicolumn{2}{|l|}{ Prospective } \\
\hline & NO CA9 MB & CA9 MB & NO CA9 MB & CA9 MB \\
\hline$s V H L$ & - & 6 & 1 & 9 \\
\hline$V H L$ & 1 & 18 & 4 & 9 \\
\hline$S D H$ & 17 & - & 20 & 2 \\
\hline TMEM127 & 1 & - & 1 & - \\
\hline sRET & 5 & - & 1 & - \\
\hline$R E T$ & 4 & - & - & - \\
\hline$s N F 1$ & 7 & 1 & 1 & - \\
\hline$N F 1$ & 5 & - & 1 & - \\
\hline$s M A X$ & 1 & 1 & - & - \\
\hline$M A X$ & 2 & - & - & - \\
\hline sHRAS & 2 & - & 5 & - \\
\hline EPAS1 & 2 & - & 4 & - \\
\hline FH/sATRX & - & 1 & - & - \\
\hline$F H$ & - & - & 2 & - \\
\hline FHs & - & - & - & 1 \\
\hline SATRX & - & 1 & - & - \\
\hline SLC25A11 & 1 & - & - & - \\
\hline$E G L N 2$ & - & - & 1 & 1 \\
\hline no mutation & 20 & 4 & 41 & 3 \\
\hline Total & 68 & 32 & 82 & 25 \\
\hline
\end{tabular}

$M B$ membrane immunostaining, $s$ somatic mutation
We considered as "negative", the tumors presenting no CA9 expression or a cytosolic labeling (Fig. 1a) and 'positive', the tumors in which we identified CA9 membrane staining, in either one or a few cells (Fig. 1b) or all tumor cells on a section (Fig. 1b). It is worth noting that sustentacular labeling was observed, and was not considered as a tumor cell positive staining (Fig. 1d).

We observed the presence of membrane CA9 labeling in 25 tumors. Among them, 5\% presented CA9 membrane staining in only 1 or 2 isolated cells on the whole tissue section, while $35 \%$ of cases exhibited between 10 and 20 isolated positive cells (representing $<1 \%$ of all cells on the slide). Focal areas of positive cells representing 1-20\% of the tissue section were seen in $35 \%$ of cases. Finally, $25 \%$ of samples showed a widespread positive membrane staining, on $80-100 \%$ of the tumor cells (Fig. 1e). Nextgeneration sequencing identified a $V H L$ variant in 18 of them ( 9 at germline level, 1 mosaic, and 8 at somatic level) (including one occurring in a germline SDHA-mutated paraganglioma) (Table 1). Among them, there were 16 different variants including 11 missense, one deletion, one splice site, one frameshift, one synonymous, and one substitution affecting the translation termination codon. Eleven of them were classified as pathogenic or likely pathogenic in the UMD-VHL database (http://www.umd.be/VHL/) and/or the ClinVar database (https://www.ncbi.nlm.nih.gov/ clinvar/?term $=$ VHL\%5Bgene\%5D) $\quad$ (Supplementary Table 1b). The germline missense variant p.(Gly144Arg) was identified in a 29 -year-old patient presenting a perirenal paraganglioma. It was previously reported in a patient with malignant pheochromocytoma [25] and was also associated with polycythemia [26]. The p.(Pro138Pro) variant was identified in a patient with a family history of VHL disease and was functionally validated by mRNA analysis, which showed a splicing alteration (data not shown). This variant was already published with evidences for pathogenicity [27]. Three of the $16 \mathrm{VHL}$ variants were not previously reported. The c.640T $>\mathrm{G}$ germline variant, leading to a protein extension with the addition of a tail of 13 new amino acids, was found in a patient affected with a single pheochromocytoma at 67 years old. The missense germline variant p.(Ala122Val) was found in a patient with bilateral
Table 2 CA9

immunohistochemistry test results predict VHL-

mutated status

\begin{tabular}{llllll}
\hline VHL gene status & Nb of tumors & NO CA9 MB & CA9 MB & Specificity & Sensitivity \\
\hline Retrospective & & & & & \\
$\quad$ VHL mutation & 25 & 1 & 24 & & $96 \%$ \\
$\quad$ No VHL mutation & 75 & 67 & 8 & $89 \%$ & \\
Prospective & & & & & $78 \%$ \\
$\quad$ VHL mutation or VUS & 23 & 5 & 18 & & \\
$\quad$ No VHL mutation & 84 & 77 & 7 & $92 \%$ & \\
\hline
\end{tabular}

VUS variants of unknown significance, $N b$ number, $M B$ membrane immunostaining 

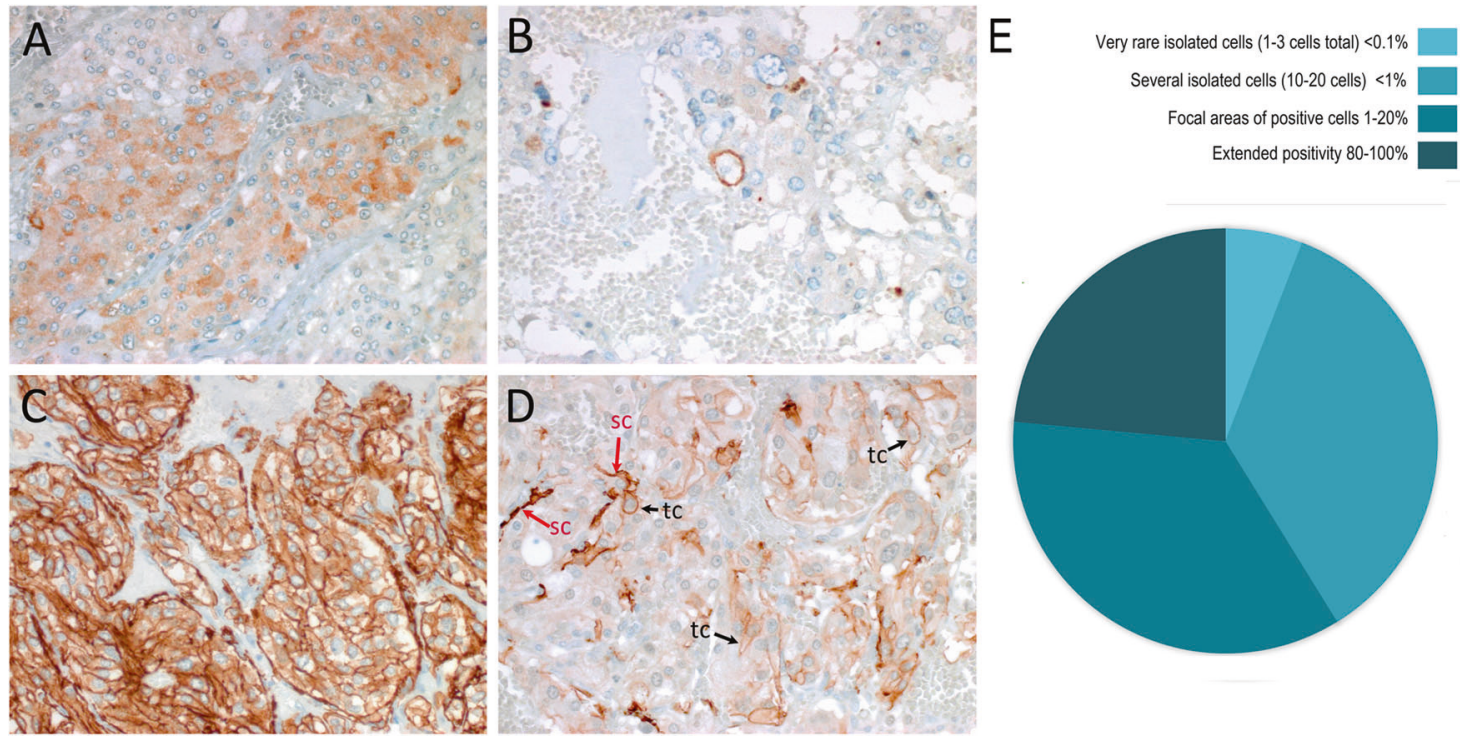

Fig. 1 CA9 protein expression in the prospective study. In non- $V H L$ mutated paraganglioma, CA9 protein expression is observed in the cytosol of tumor cells (a), while membrane staining of tumor cells (black arrows) was only found in $V H L$-mutated paraganglioma (b-d). Sustentacular staining should not be considered as a tumor membrane

labeling (red arrows in D). The different samples were classified in four groups depending on the distribution of CA9 staining from $0.1 \%$ to almost $100 \%$ of tumor cells. The proportion of tumors classified in each group is shown in $\mathbf{e}$

pheochromocytoma diagnosed at 47 years old. The p. Val87Glu somatic variant was identified with an allelic ratio of $8 \%$ in a pheochromocytoma developed by a 29 -year-old patient.

Among the seven tumors with CA9 membrane immunostaining in which no $V H L$ variant could be identified, two were head and neck paraganglioma, one without any mutation detected in genes included in the "MASTR Plus SDHv2" next-generation sequencing panel, one with a likely pathogenic variant in SDHD gene (c335_341delinsTTGACTC), p.(Thr112_Tyr114delinsIleAspSer) associated with a negative SDHB immunostaining. Three were abdominal paragangliomas, a pelvic paraganglioma carrying biallelic $F H$ somatic mutations and two pheochromocytomas, one with a synonymous EGLN2 somatic variant, and one without any germline or somatic variant. Finally, two patients developed multiple paraganglioma, one with an $S D H D$ germline mutation, and one for whom the somatic next-generation sequencing analyses assessed in two different tumors were negative.

Eighty-two samples showed no CA9 membrane staining (Table 1). In these patients/tumors, next-generation sequencing did not identify any mutation in 41 cases but detected 20 SDHx mutations (8 SDHA, 7 SDHB, 4 SDHC, and $1 S D H D)$, 5 somatic HRAS mutations, 4 EPAS1 somatic mutations, 2 NF 1 mutations (1 germline and 1 somatic), 2 FH mutations, 1 somatic RET mutation, 1 TMEM127, and 1 EGLN2 variant of unknown significance. Five patients carried a germline $(n=4)$ or a somatic $V H L$ variant $(n=1)$. For two of them (one germline (patient \#84) and the somatic case (patient \#45)), we were able to retrieve two and three additional blocks, respectively. In the first patient, we identified five and two CA9-positive cells in the two additional blocks, respectively. In the second, two of the supplementary blocks showed one and two positive cells, respectively, while we were not able to find any positive cell in the third block. Because the evaluation of several blocks was not performed in all cases, these results were however not included in the calculation of performance.

Hence these prospective data revealed that CA9 immunohistochemistry allowed detecting $V H L$ mutations with a specificity of $92 \%$ and sensitivity of $78 \%$ when evaluating only one block of each tumor (Table 2).

\section{Discussion}

In the current study, we evaluated CA9 expression as a tool to predict or validate $V H L$ gene mutations in paragangliomas. We show that the presence of a CA9 immunostaining specifically localized at the plasma membrane of tumors cells is a specific and sensitive criterion that is associated with a VHL-mutated status. Altogether, CA9 membranous labeling was observed in 42/48 (88\%) VHL tumors and was absent in 144/159 (91\%) non-VHL tumors in this study.

The impact of genetic determinism is crucial in paraganglioma. Indeed, these tumors represent the human tumors, for which the proportion of germline mutations in predisposing genes is the most important. There are now more than 15 paraganglioma susceptibility genes described, 
some of them being also mutated at somatic level, in sporadic forms of these tumors. The Endocrine Society clinical practice guidelines have recommended that "all patients with paraganglioma should be engaged in shared decision making for genetic testing" [28] and it was recently showed that the identification of a mutation in an $S D H x$ or $V H L$ gene has a direct and a positive impact on the management and the clinical outcome of the affected patients [29]. Depending on the centers, sequencing of the paraganglioma susceptibility genes may be performed by the former Sanger method, or by next-generation sequencing with target gene panels [24, 30, 31], for which an international consensus statement has been published [8]. Whatever the techniques, orientation of the genetic test or validation of variants of unknown significance is of critical importance to offer the best clinical management to patients affected by a hereditary disease and to their relatives. Several immunohistochemical staining have therefore been developed and validated to help with interpretation of genetic variants or to detect mutated tumors. In particular, SDHB and SDHA immunohistochemistries are now used worldwide and acknowledged by the international guidelines for the management of SDH-related patients [28]. Following SDHx mutations, VHL mutations are the second cause of inherited paraganglioma [8] and are also frequently identified at somatic level [22]. However, no biomarker was available and validated to predict their presence or validate their pathogenicity. Surprisingly, although CA9 is expected to be transcriptionally regulated by pseudohypoxia, overexpression of its mRNA was not a reproducible hallmark of VHL-mutated tumors. Indeed, among the 40 VHL-mutated tumors for which we had generated transcriptomic data, 16 displayed a sometimes-massive overexpression of CA9 while the remaining 24 had CA9 mRNA levels similar to other genotypes. Interestingly, $S D H$-mutated tumors, which have also been demonstrated to display a pseudohypoxic signature, did not show any increase in CA9 expression. Hence, although CA9 high mRNA levels were solely observed in $V H L$-mutated tumors, they could not be considered as a transferable tool for $V H L$ status prediction. Evaluation of CA9 protein expression by immunohistochemistry on FFPE tissues confirmed that a quantitative estimation would not be relevant as very heterogeneous staining were observed inter and intra-tumors. In the contrary, qualitative analysis of CA9 protein localization is a very strong tool to study $V H L$-related paraganglioma. Interestingly, Pinato et al. reported a study that comprised four VHL germline mutated cases in which they also observed a predilection for strong membranous CA9 staining [20]. To the best of our knowledge, this study is the first one to propose an immunohistochemical tool that could be used in routine pathology practice to detect VHLmutated paraganglioma. Because of the high frequency of germline and somatic $V H L$ mutations we propose that CA9 immunohistochemistry could be performed simultaneously to SDHB immunohistochemistry in all paragangliomas and be associated with $V H L$ germline (and if possible somatic) genetic testing. The major weakness of this biomarker is that the number of CA9 positive cells is highly variable between samples, from almost all tumor cells within a tumor section (in $25 \%$ of cases) to a single positive cell within the whole section (in $5 \%$ of cases). The reason for this heterogeneity is unexplained and it was neither associated with the type of mutation (germline or somatic) nor with a specific mutation. It may be caused by a specific condition of the tumor microenvironment or by local $\mathrm{pH}$ differences. Anyhow, the cases where the number of cells was very low obviously reveal the limit of this marker. They shed light on the fact that CA9 staining should be searched for carefully, on tissue sections as large as possible, and not on biopsies (which are anyway almost never performed for paraganglioma [32]). In two cases, we have also shown that the use of several blocks of a single tumor may reveal positive cells in suspiciously negative cases.

\section{Conclusion}

To date, CA9 immunohistochemistry is the first immunohistochemical tool that reliably helps in identifying or validating $V H L$ genetic variants and our data suggest that it should be added to the portfolio of antibodies currently used for analyzing paraganglioma in routine pathology practice.

Acknowledgements We are grateful to Caroline Travers, Valentin Adamus, Nelly Le Pottier, Mathieu Madelaine, Karine Auribault, Patrick Nitschke, and Annabelle Venisse for their technical contribution to this work as well as Catherine Tritscher for administrative assistance. We thank the Biological Resources Center and Tumor Bank Platform, Hôpital Européen Georges Pompidou (BB-003300063). We are specifically grateful to the following pathologists, investigators of the COMETE-TACTIC consortium, who provided tumor tissues as well as their local biological resources centers: Dr Abir Ghuzlan (Gustave Roussy, Villejuif), Dr Frédérique Tissier (APHP, Hôpital de la Pitié Salpétrière, Paris), Dr Sophie Michalak (CHU Angers), Dr Valérie Coste-Martineau (Hôpital Arnaud de Villeneuve, CHU Montpellier), Dr Yves Allory (AP-HP, Hôpital Henri Mondor, Créteil), Pr Catherine Guettier-Bouttier (AP-HP, Hôpital de Bicêtre, Le Kremlin-Bicêtre), Pr Jean-Philippe Merlio (CHU Bordeaux), Dr Karine Renaudin (CHU Nantes), Pr Nathalie Sturm (CHU Grenoble), Dr Myriam Decaussin-Petrucci (CHU Lyon), Dr Serge Guyetant (CHU Tours), Dr Danielle Diebold (CHU de Reims), Pr Nathalie RiouxLeclercq (CHU Rennes), Pr Marie-Pierre Neu-Chenard (CHU Strasbourg), and Pr Anne Gomez-Brouchet (CHU Toulouse).

Funding This work has received funding from the European Union's Horizon 2020 research and innovation program under grant agreement no 633983 and by the Institut National du Cancer and the Direction Générale de l'Offre de Soins (PRT-K 2014, COMETE-TACTIC, INCa-DGOS_8663). NB received a financial support from the Cancer Research for Personalized Medicine-CARPEM project (Site de 
Recherche Intégré sur le Cancer-SIRIC). Our team is supported by the Ligue Nationale contre le Cancer (Equipe Labellisée).

\section{Compliance with ethical standards}

Conflict of interest The authors declare that they have no conflict of interest.

Ethics All patients provided written informed consent for paraganglioma genetic testing, collection of samples, and subsequent analyses. Ethical approval for the study was obtained from the institutional review board (IRB 00003835, Comité de Protection des Personnes Ile de France IV, September 2015). This work is a part of the COMETE-TACTIC study (ClinicalTrials.gov Identifier: NCT02672020).

Publisher's note: Springer Nature remains neutral with regard to jurisdictional claims in published maps and institutional affiliations.

\section{References}

1. Dahia PL. Pheochromocytoma and paraganglioma pathogenesis: learning from genetic heterogeneity. Nat Rev Cancer. 2014;14:108-19.

2. Favier J, Amar L, Gimenez-Roqueplo AP. Paraganglioma and phaeochromocytoma: from genetics to personalized medicine. Nat Rev Endocrinol. 2015;11:101-11.

3. Remacha L, Comino-Mendez I, Richter S, Contreras L, CurrasFreixes M, Pita G, et al. Targeted exome sequencing of Krebs cycle genes reveals candidate cancer-predisposing mutations in pheochromocytomas and paragangliomas. Clin Cancer Res. 2017;2:6315-24.

4. Calsina B, Curras-Freixes M, Buffet A, Pons T, Contreras L, Leton $\mathrm{R}$, et al. Role of $\mathrm{MDH} 2$ pathogenic variant in pheochromocytoma and paraganglioma patients. Genet Med. 2018;20:1652-62.

5. Remacha L, Curras-Freixes M, Torres-Ruiz R, Schiavi F, TorresPerez R, Calsina B, et al. Gain-of-function mutations in DNMT3A in patients with paraganglioma. Genet Med. 2018;20:1644-51.

6. Buffet A, Morin A, Castro-Vega LJ, Habarou F, Lussey-Lepoutre $\mathrm{C}$, Letouze $\mathrm{E}$, et al. Germline mutations in the mitochondrial 2oxoglutarate/malate carrier SLC25A11 gene confer a predisposition to metastatic paragangliomas. Cancer Res. 2018;78:1914-22.

7. Castro-Vega LJ, Lepoutre-Lussey C, Gimenez-Roqueplo AP, Favier J. Rethinking pheochromocytomas and paragangliomas from a genomic perspective. Oncogene. 2016;35:1080-9.

8. Toledo RA, Burnichon N, Cascon A, Benn DE, Bayley JP, Welander J, et al. Consensus statement on next-generationsequencing-based diagnostic testing of hereditary phaeochromocytomas and paragangliomas. Nat Rev Endocrinol. 2017;13:233-47.

9. Ayala-Ramirez M, Chougnet CN, Habra MA, Palmer JL, Leboulleux S, Cabanillas ME, et al. Treatment with sunitinib for patients with progressive metastatic pheochromocytomas and sympathetic paragangliomas. J Clin Endocrinol Metab. 2012;97:4040-50.

10. Jimenez C, Cabanillas ME, Santarpia L, Jonasch E, Kyle KL, Lano EA, et al. Use of the tyrosine kinase inhibitor sunitinib in a patient with von Hippel-Lindau disease: targeting angiogenic factors in pheochromocytoma and other von Hippel-Lindau disease-related tumors. J Clin Endocrinol Metab. 2009;94:386-91.

11. van Nederveen FH, Gaal J, Favier J, Korpershoek E, Oldenburg RA, de Bruyn EM, et al. An immunohistochemical procedure to detect patients with paraganglioma and phaeochromocytoma with germline SDHB, SDHC, or SDHD gene mutations: a retrospective and prospective analysis. Lancet Oncol. 2009;10:764-71.

12. Burnichon N, Briere JJ, Libe R, Vescovo L, Riviere J, Tissier F, et al. SDHA is a tumor suppressor gene causing paraganglioma. Hum Mol Genet. 2010;19:3011-20.

13. Korpershoek E, Favier J, Gaal J, Burnichon N, van Gessel B, Oudijk $\mathrm{L}$, et al. SDHA immunohistochemistry detects germline SDHA gene mutations in apparently sporadic paragangliomas and pheochromocytomas. J Clin Endocrinol Metab. 2011;96:E1472-6.

14. Menara M, Oudijk L, Badoual C, Bertherat J, Lepoutre-Lussey C, Amar L, et al. SDHD immunohistochemistry: a new tool to validate SDHx mutations in pheochromocytoma/paraganglioma. $\mathrm{J}$ Clin Endocrinol Metab. 2015;100:E287-91.

15. Papathomas TG, Oudijk L, Persu A, Gill AJ, van Nederveen F, Tischler AS, et al. SDHB/SDHA immunohistochemistry in pheochromocytomas and paragangliomas: a multicenter interobserver variation analysis using virtual microscopy: a Multinational Study of the European Network for the Study of Adrenal Tumors (ENS@T). Mod Pathol. 2015;28:807-21.

16. Castro-Vega LJ, Buffet A, De Cubas AA, Cascon A, Menara M, Khalifa E, et al. Germline mutations in FH confer predisposition to malignant pheochromocytomas and paragangliomas. Hum Mol Genet. 2014;23:2440-6.

17. Burnichon N, Cascon A, Schiavi F, Morales NP, CominoMendez I, Abermil N, et al. MAX mutations cause hereditary and sporadic pheochromocytoma and paraganglioma. Clin Cancer Res. 2012;18:2828-37.

18. Vogel TW, Vortmeyer AO, Lubensky IA, Lee YS, Furuta M, Ikejiri $\mathrm{B}$, et al. Coexpression of erythropoietin and its receptor in endolymphatic sac tumors. J Neurosurg. 2005;103:284-8.

19. Nakamura E, Abreu-e-Lima $P$, Awakura $Y$, Inoue $T$, Kamoto $T$, Ogawa $\mathrm{O}$, et al. Clusterin is a secreted marker for a hypoxiainducible factor-independent function of the von Hippel-Lindau tumor suppressor protein. Am J Pathol. 2006;168:574-84.

20. Pinato DJ, Ramachandran R, Toussi ST, Vergine M, Ngo N, Sharma R, et al. Immunohistochemical markers of the hypoxic response can identify malignancy in phaeochromocytomas and paragangliomas and optimize the detection of tumours with VHL germline mutations. Br J Cancer. 2013;108:429-37.

21. Al-Ahmadie HA, Alden D, Qin LX, Olgac S, Fine SW, Gopalan A, et al. Carbonic anhydrase IX expression in clear cell renal cell carcinoma: an immunohistochemical study comparing 2 antibodies. Am J Surg Pathol. 2008;32:377-82.

22. Burnichon N, Vescovo L, Amar L, Libe R, de Reynies A, Venisse A, et al. Integrative genomic analysis reveals somatic mutations in pheochromocytoma and paraganglioma. Hum Mol Genet. 2011;20:3974-85.

23. Castro-Vega LJ, Letouze E, Burnichon N, Buffet A, Disderot PH, Khalifa E, et al. Multi-omics analysis defines core genomic alterations in pheochromocytomas and paragangliomas. Nat Commun. 2015;6:6044.

24. Ben Aim L, Pigny P, Castro-Vega LJ, Buffet A, Amar L, Bertherat $\mathrm{J}$, et al. Targeted next-generation sequencing detects rare genetic events in pheochromocytoma and paraganglioma. J Med Genet. 2019;56:513-20.

25. Yates CJ, McAuley SA, Grodski S, Hamblin PS, Ebeling PR. An elusive phaeochromocytoma. Med J Aust. 2011;194:44-5.

26. Nordstrom-O'Brien M, van der Luijt RB, van Rooijen E, van den Ouweland AM, Majoor-Krakauer DF, Lolkema MP, et al. Genetic analysis of von Hippel-Lindau disease. Hum Mutat 2010;31:521-37.

27. Lenglet M, Robriquet F, Schwarz K, Camps C, Couturier A, Hoogewijs D, et al. Identification of a new VHL exon and complex splicing alterations in familial erythrocytosis or von HippelLindau disease. Blood. 2018;132:469-83. 
28. Lenders JW, Duh QY, Eisenhofer G, Gimenez-Roqueplo AP, Grebe SK, Murad MH, et al. Pheochromocytoma and paraganglioma: an endocrine society clinical practice guideline. J Clin Endocrinol Metab. 2014;99:1915-42.

29. Buffet A, Ben Aim L, Leboulleux S, Drui D, Vezzosi D, Libe R, et al. Positive impact of genetic test on the management and outcome of patients with paraganglioma and/or pheochromocytoma. J Clin Endocrinol Metab. 2019;104:1109-18.

30. Curras-Freixes M, Pineiro-Yanez E, Montero-Conde C, Apellaniz-Ruiz M, Calsina B, Mancikova V, Remacha L, et al.
PheoSeq: a targeted next-generation sequencing assay for pheochromocytoma and paraganglioma diagnostics. J Mol Diagn. 2017;19:575-88.

31. Toledo RA, Dahia PL. Next-generation sequencing for the diagnosis of hereditary pheochromocytoma and paraganglioma syndromes. Curr Opin Endocrinol Diabetes Obes. 2015;22: 169-79.

32. Vanderveen KA, Thompson SM, Callstrom MR, Young WF Jr., Grant CS, Farley DR, et al. Biopsy of pheochromocytomas and paragangliomas: potential for disaster. Surgery. 2009;146:1158-66. 